\title{
Mesh Simplification Using Vertex Clustering Based on Principal Curvature
}

\author{
Zhen Hua, Zilong Huang and Jinjiang Li \\ Shandong Institute of Business and Technology, Yantai, China \\ Shandong Normal University, Jinan, China \\ Shandong Institute of Business and Technology, Yantai, China \\ huazhen66@foxmail.com
}

\begin{abstract}
According to the problem that how to increase the fidelity of simplified model, this paper put forward a method which using vertex clustering based on principal curvature. Our measure taking the gradual simplify thinking and simplifying the mesh model successive by quadric error metric and principal curvature based on integral invariants. We improve the defect of error accumulation which consisted in the previous algorithms. Not only ensuring the display effect for the model, but also raising the efficiency of algorithm.
\end{abstract}

Keywords: 3D mesh simplification, vertex clustering, triangle mesh, quadric error metric; integral invariants, principal curvature

\section{Introduction}

With the development of computer technology and laser scanning equipments constantly, the 3D models become complex and detailed which applied to computer graphics, computer aided geometric design, virtual reality, reverse engineering and other field. In general, the 3D models are represented by triangle mesh from reconfiguration (polygon mesh can be converted into triangle mesh through triangulated, so, the research for triangle mesh models have general meaning). The models has become more and more detailed, it means that the number of triangle patches which constitute the mesh model has up to millions or even billions as usual. Due to the positive correlation between the number of triangle patches and the cost of processing them, the storage, real-time rendering and delivery for mass information of models has brought an unprecedented challenge for computer hardware and network transmission.

Every category of mesh model simplification methods has its own characteristic. According to the difference of executing state for simplification, we can divide the simplification algorithms into two kinds, static and dynamic. And also can from the viewpoint whether the topology structure has changed in the simplification process, divided into topology preserving and topology change. However, these diverse classifications are hard to contain all the simplification algorithms that cross each other. The essence of model simplification is actually using appropriate methods to 
minimize the number of vertices, edges and patches, while maintaining the geometrical characteristics property of original model. In other words, that is ensuring certain accuracy, at same time, to reduce the scale of data as much as possible. In this process, how to improve the efficiency and control the simplification error have attracted a lot of attention from domestic and foreign scholars [1]. This paper presents a new vertex clustering simplification method based on principal curvature. Our method choose the principal curvature of vertex as characteristic, using the characteristic points as less as possible to represent the detailed information of original model as much as possible and keep the loss of local visual characteristic to a minimum. And it also reflect the two general principles of model simplification, first, the least number of vertices principle, second, the minimal difference principle which between original model and simplified model. The precondition of all those two principles is error in the controllable range.

Firstly, according to the different operational objects in the process of model simplification, this paper summarizes the current situation and research achievements of 3D mesh model simplification; secondly, making a detailed introduction of basic concepts, related principles and the main steps which proposed in our paper; finally, verifying the feasibility of this method, analyzing the superiority that compared with the similar algorithms. To summarize the content which needed to supplement and improve.

\section{Related Work}

In recent years, there are a lot of fruitful researches in the mesh model simplification, while most of them are working based on the geometrical elements that removed in the process of simplification, such as vertex deletion, edge collapse and patch combination.

As a widely used mesh model simplification algorithm which based on vertex clustering, it is easiest to achieve and has the highest efficiency, but the simplification result can't be guarantee is one of the main drawbacks [2]. Rossignac proposed a simplification method which is faster and easy to operate based on vertex clustering, nevertheless, this method has some limitations: on one hand, due to it didn't take the vertex distribution into consideration, the algorithm resulted in wasting time and space, on the other hand, there is no method given to control the errors when we determine the new vertices [3]. Zhou et al., made a self-adaptable partition on bounding box of mesh model by using the Octree, and put forward an error control method based on the distance from point to surface. Compared with Rossignac's algorithm, applied extensively and obvious effects are both the advantages [4]. Turk presented a mesh re-tiling simplification algorithm based on positioning vertices, the method distributes a certain number of vertices on the original mesh model by using the point repulsion. And then, using the new vertices and old vertices to structure a middle mesh, finally, deleting the old vertices and re-triangulated to get the simplified model. The limitations of this method concentrate on the distribution of initial vertices. Only using the point repulsion as a reference is not enough to make a better result on the models which has the rough 
surface, and also increasing the error because of its huge calculation [5]. After taking a sufficient analysis for the defects of point repulsion, Zhou and Ma applied the curvature and area of triangle as essential factors to reflect the characteristic information of mesh model, made a cross-consider on importance degree of these two factors and designed a new positioning vertices algorithm, got fine results [6].

Hoppe first proposed the edge collapse simplification algorithm that applied to the two-dimensional manifold of triangle mesh models. To measure the original mesh with an energy function, they established the complex equation for optimizing global energy. The non-linearization of optimization procedure for mesh model has led to huge calculation and low arithmetic speed [7]. In order to overcome these shortcomings, Tao and Pan introduced the energy value to evaluate the meta operation and adjust the matching relation between original mesh and simplified mesh. Their algorithm not only be applied to arbitrary topological shape, but also can change itself according to different requirements [8]. In order to control the error effectively and increase the accuracy of simplification, Zhou et al. aimed at the surface reconstruction of reverse engineering, proposed a new algorithm that has a high degree of automation and no error accumulation by combining the controlling based on examining ball with edge deletion [9]. In the process of edge collapse, Kibbelt et al. regarded one of the endpoints on the edge as the representative vertex instead of adding a new vertex. This method improves the efficiency of rendering while using less memory [10].

The scientists also concentrate on the deletion and combination of triangle patch in the mesh model simplification algorithms. Hamann calculated the error for simplification based on the curvature and equal angle of the triangle. He regarded the mean of curvatures for vertices as weight, removed the triangles which long and narrow. Although it is easy to operate, it has the huge calculation [11]. Gieng et al. proposed a similar triangle collapse algorithm. The difference is that the latter regarded the product of area and curvature as the cost of collapse. This change can maintain the shape characteristics preferably, but it costs the algorithmic complexity [12]. According to the method presented by Hamann, Ma et al. improved its triangle removal criterion to decrease the whole calculation and preserve the feature availably [13]. Zhou et al. described the square volume error, shape factor and normal constraint factor as a quadratic objective function, afterwards, put forward a new triangle collapse simplification algorithm which can keep the boundary feature effectively [14].

Another important branch of mesh simplification is dynamic deformation mesh simplification. Shamir presented a global multi-resolution structure based on time-dependant directed acyclic graph. It is the first simplification algorithm for distorted surface, but the data structure is too complex to control $[15,16]$. Kircher et al. regarded the successive edge collapse as re-clustering. To obtain the simplified model for current frame, they used the vertices exchange operations of previous frame. The multi-resolution method which has a dynamic connectivity, not only aims at distorted surface but also with high efficiency [17]. In order to get the perfect 
effect, Huang applied the vertex tree to change the dynamic connectivity, this operation increases the quality of reproduction, but it also brings huge calculation and complex process [18].

There are many scholars considered simplifying the models from multiple angles and obtained some better results. Lv, Zhang and Sun collapsed the edge preferentially which has less error after collapsed and got a coarse mesh with good topological connectivity. Then, simplify the coarse mesh through introducing new vertices with good valance. The method greatly increases the quality of simplified mesh [19]. Cohen-Steiner et al. divided the original mesh into several clusters of similar surfaces and optimized them via the algorithm of Lloyd. They gained the simplified mesh by using polygons to represent every cluster. Although the algorithm has preferable stability, however, due to the time of iteration is unknown, it is difficult to improve its speed [20].

In the field of virtual reality, the original model contains not only the geometric properties, but also a lot of texture, color and other information. How to integrate the characteristic information into the process of simplification is the popular research in recent years. In order to solve the discontinuities of appearance attributes in the simplification, Lu, Zeng and Pan proposed a new formula for the cost of edge collapse by analyzing the relationship between the discrete appearance seam and vertices, and then, they were applying the half-edge collapse to improve the QEM. Their algorithm has a good performance in feature-preserving [21]. Zhang et al. used the symmetric Hausdorff distance in $R^{n}$ space to control the order of error and accuracy of simplification. The algorithm can preserve the additional information of color and texture [22]. Liu, Xie and Jin aimed at the mesh models which have texture attributes, then marked the boundary edges and texture edges of the model in advance, weighted them according to the importance degree. Furthermore, they combined the half-edge collapse with weight in the simplification to confirm the order of collapse and get the simplified model [23]. According to the calculation for geometrical error based on QEM, Feng and Zhou added the texture error calculation in the former. The algorithm retains the texture of surface for the model successfully [24].

\section{Description of the Algorithm}

This paper take a hierarchical and gradual simplify mode. First, we classify and choose the vertices of mesh model preliminarily, delete the redundant vertices which have similar characteristic. And then, according to the principal curvature, we get the final representative vertices through conducting the cluster on the remaining vertices which have more important degree for the simplified model.

\subsection{Model Preprocessing}

Due to the stability of triangle patch, there is an irreplaceable advantage of triangle mesh for indicating the free-form surface and drawing the 3D models. And it 
also becomes the mainly method for representing the 3D models in the field of interactive computer graphics.

Under ordinary circumstances, 3D model mostly represented by polygon mesh, and the polygon mesh can divide into several triangles by connecting the vertices. With this operation, we can triangulate every surface of the pending polyhedral model to get the triangle mesh. We numbered all those triangles and recorded them in triangle table $T$. Figure 1 shows an example of dividing a polygon into several triangles.
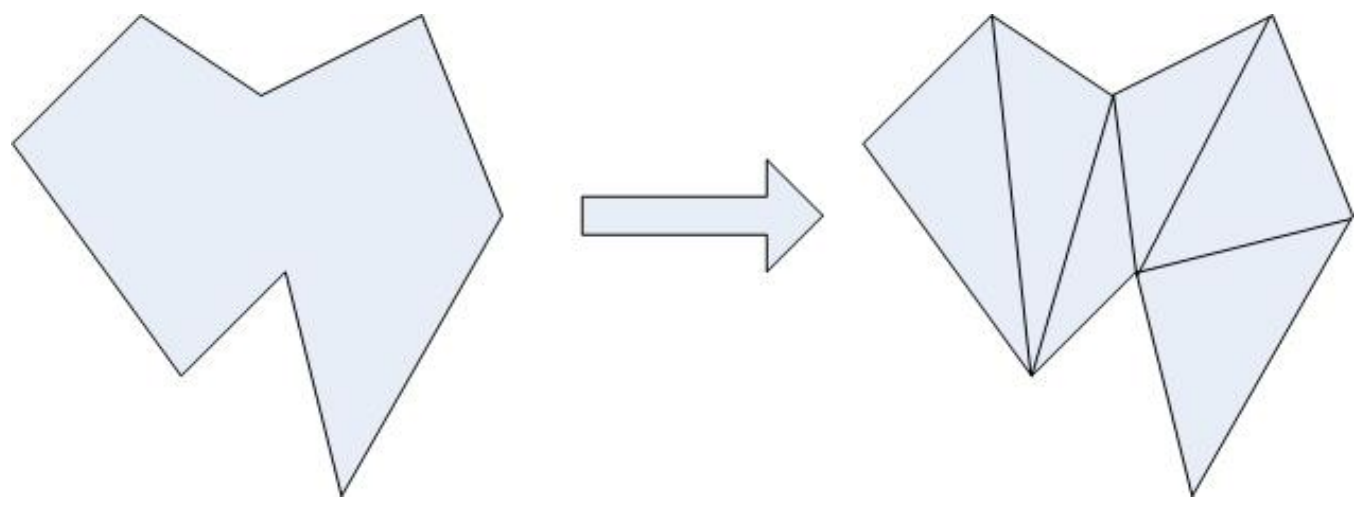

Figure 1. The Way to Divide Polygons

\subsection{The Classical QEM Algorithm}

Garland first presented the concept of QEM and made it as the standard for measuring the cost of edge collapse. The algorithm defined the square of distance from point to surface as the error to control the simplified precision of models. It has high efficiency and minimal occupancy of computer memory [25].

In the range of Euclidean geometry, define a vertex:

$$
p=\left[\begin{array}{llll}
p_{x} & p_{y} & p_{z} & 1
\end{array}\right]^{T}
$$

Make

$$
f=\left[\begin{array}{llll}
a & b & c & d
\end{array}\right]^{T}
$$

To indicate the triangle patch:

$$
\left\{\begin{array}{l}
a x+b y+c z+d=0 \\
a^{2}+b^{2}+c^{2}=1
\end{array}\right.
$$

The normal vector is:

$$
\vec{n}=(a, b, c)^{T}
$$

Then the quadratic form of error for $P$ is:

$$
\Delta p=p^{T} Q p=\Delta\left(\left[\begin{array}{llll}
p_{x} & p_{y} & p_{z} & 1
\end{array}\right]^{T}\right)=\sum_{p \in F}\left(p^{T} f\right)^{2}
$$

$F$ is a set of triangles which both take the $p$ as their own vertex. By further 
rewriting, we can get another representation like:

$$
\Delta p=\sum_{p \in F}\left(p^{T} f\right)\left(f^{T} p\right)=\sum_{p \in F} p^{T}\left(f f^{T}\right) p=p^{T}\left(\sum_{p \in F} K_{f}\right) p
$$

$K_{p}$ is used to determine the square of distance from a certain vertex to $f$,

$$
K_{p}=f f^{T}\left[\begin{array}{llll}
a^{2} & a b & a c & a d \\
a b & b^{2} & b c & b d \\
a c & b c & c^{2} & c d \\
a d & b d & c d & d^{2}
\end{array}\right]
$$

Make the sum of $K_{p}$ for all neighborhood triangles which use the $p$ as their own vertex. In that way, we can get the value of error.

\subsection{Preliminary Classify and Simplify for Vertices Based on Quadric Error Metric}

Step 1: Apply the bounding box to surround the original mesh model, subdivide the bounding box into $N$ cells averagely based on its edges. When some vertices into the same cell, they can form a certain class. We numbered all those classes and recorded them in vertex index table $N$.

Step 2: When one triangle's three vertices all belong to the same class which in the triangle table $T$, these three vertices degenerate into a new point, the point is located in the centre of gravity.

Step 3: When a triangle's two vertices belong to the same class, we apply the QEM to calculate the value of error for the two vertices, and then, remain the vertex which has less value than the other one. Figure 2 shows that how to combine vertices when two vertices belong to the same class.
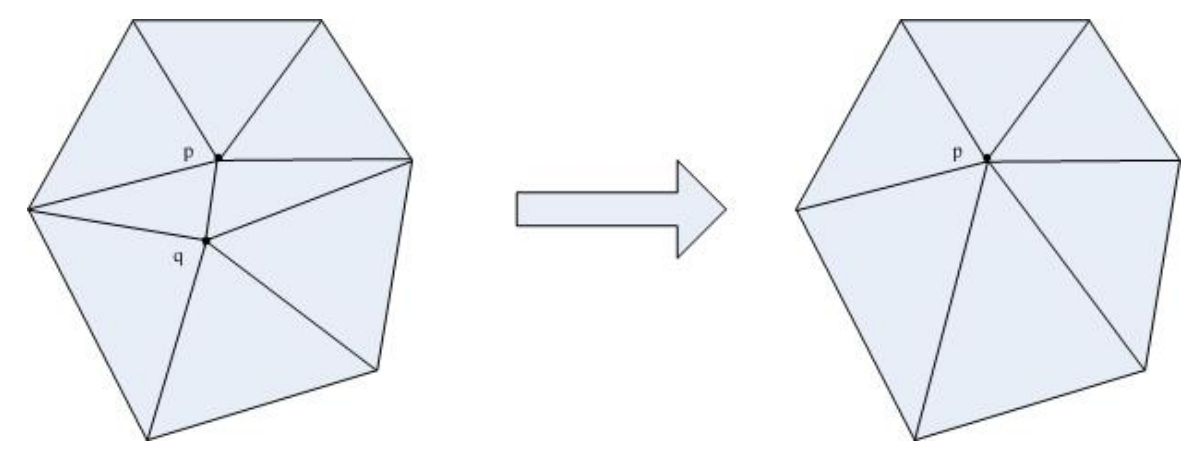

Figure 2. The Error Value of $p$ is Less than that of $q$, so Delete the $q$

Step 4: When the class of a vertex is different from other two vertices in one triangle, keep this vertex intact.

Through the above several steps of vertex clustering and delete, we have already eliminated the redundant vertices which can represent the same characteristic 
information in the mesh model. These operations decrease the space occupancy and improve the efficiency of whole algorithm; they also reduce the time complexity and provide advantage for the further simplification.

\subsection{The Selection Algorithm of Representative Vertices Based on Principal Curvature}

Although doing a series of preliminary simplification, there are still many untreated redundant vertices in the mesh model due to the simple classification. We need improving the classification to further simplify the vertices. Owing to the principle curvature of a vertex on the surface can reflect the geometrical characteristics more than other elements. And also the curvature is insensitive to the noise interference so that it has better robustness. This is the reason why we apply the principle curvature to obtain the representative vertices.

Firstly, we apply the integral invariants to estimate the principal curvature for every cell among the bounding box that used by preliminary simplification. Assuming a point set $\mathrm{Q}$ is a small set near the point $\mathrm{p}$ which radius is $\mathrm{r}$. We calculate the volume of the point set Q[26, 27]:

$$
V_{Q} \int_{Q} d x
$$

Secondly, calculating the gravity centre of its:

$$
S_{Q}=\frac{1}{V_{Q}} \int_{Q} x d x
$$

then, obtaining its covariance matrix:

$$
J_{Q} \int_{Q} x x^{T} d x-V_{Q} S_{Q} S_{Q}^{T}
$$

Where $X X^{T}$ is a $3 \times 3$ matrix has the rank is 1 (We use the column vector). If $\mathrm{Q}$ is the ball neighborhood, according to the formulae above, we can get the eigenvalues of the covariance matrix for Q:

$$
\begin{aligned}
& M_{b, 1}^{r}=\frac{2 \pi}{15} r^{5}-\frac{\pi}{48}\left(3 k_{1}+k_{2}\right) r^{6}+O\left(r^{7}\right) \\
& M_{b, 2}^{r}=\frac{2 \pi}{15} r^{5}-\frac{\pi}{48}\left(k_{1}+3 k_{2}\right) r^{6}+O\left(r^{7}\right)
\end{aligned}
$$

When $r$ gets close to 0 , we can calculate the two principal curvature values by formula (13) and formula (14):

$$
\begin{gathered}
K_{b, 1}^{r}=\frac{6\left(M_{b, 2}^{r}-3 M_{b, 1}^{r}\right)}{\pi r^{6}}+\frac{8}{5 r} \\
K_{b, 2}^{r}=\frac{6\left(M_{b, 1}^{r}-3 M_{b, 2}^{r}\right)}{\pi r^{6}}+\frac{8}{5 r}
\end{gathered}
$$

If $\mathrm{Q}$ is the spherical neighborhood, similarly, gaining the formula (15) and 
formula (19):

$$
\begin{aligned}
& M_{s, 1}^{r}=\frac{2 \pi}{3} r^{4}-\frac{\pi}{8}\left(3 k_{1}+k_{2}\right) r^{5}+O\left(r^{6}\right) \\
& M_{s, 2}^{r}=\frac{2 \pi}{3} r^{4}-\frac{\pi}{8}\left(k_{1}+3 k_{2}\right) r^{5}+O\left(r^{6}\right)
\end{aligned}
$$

The two principal curvature values:

$$
\begin{gathered}
K_{s, 1}^{r}=\frac{\left(M_{s, 2}^{r}-3 M_{s, 1}^{r}\right)}{\pi r^{5}}+\frac{4}{3 r} \\
K_{s, 2}^{r}=\frac{\left(M_{s, 1}^{r}-3 M_{s, 2}^{r}\right)}{\pi r^{5}}+\frac{4}{3 r}
\end{gathered}
$$

Secondly, comparing the principal curvature of every cell with the principle curvature of vertices in it, and then choose the vertices which principle curvature is equal with its own cells respectively, regard them as representative vertices. If there is no vertex to conform to this condition, go on subdividing the bounding box until finding the representative vertices by iterative algorithm.

Finally, after all the representative vertices are found, we can get the simplified mesh model by triangulated the new vertices.

\section{Experiments}

When we take the experiments, we choose the representative vertices based on general curvature and principle curvature respectively for contrast. Figure 3 provide the selected results of representative vertices and their corresponding simplified models. The first column is based on general curvature; the second is based on principle curvature, the last two columns show the simplified models correspond to the first and second.

According to the comparison between $\mathrm{a}$ and $\mathrm{b}$ of the face models, the number of representative vertices chose by the general curvature is more than using the principle curvature on the same parts which have remarkable characteristics. However, in ear positions, because of the surface undulation is less prominent, so that the number of representative vertices has not much difference. It is obvious that the feature extraction based on principle curvature is better than the general curvature in the visual effect from comparing $\mathrm{c}$ with $\mathrm{d}$.

Similarly, the general curvature method needs a lot of representative vertices to represent the local positions that have rich lines like the legs of horse. In other words, the principle curvature method not only reduces the computational scale, but also the simplified efficiency exceeds the former. 


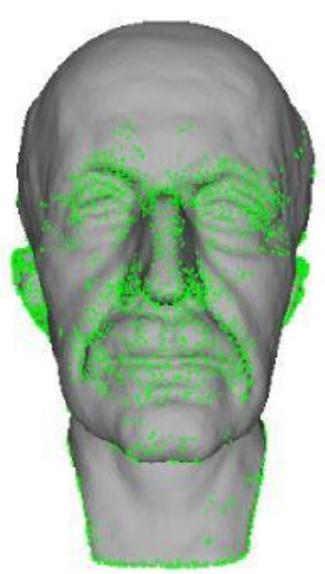

(a)



(a)

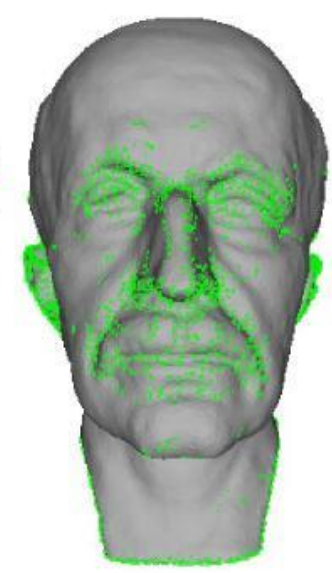

(b)

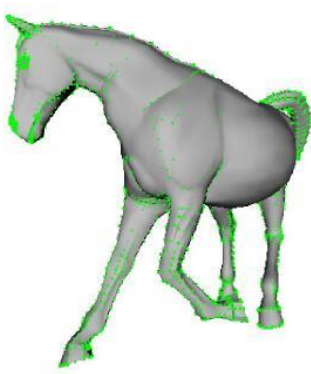

(b)

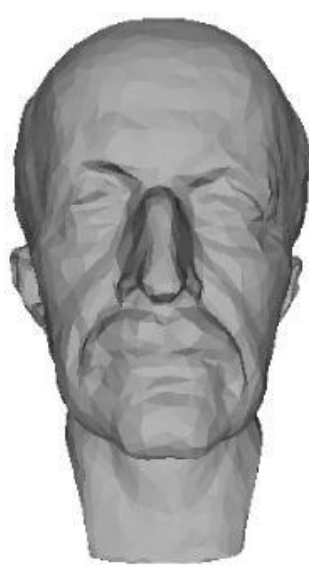

(c)

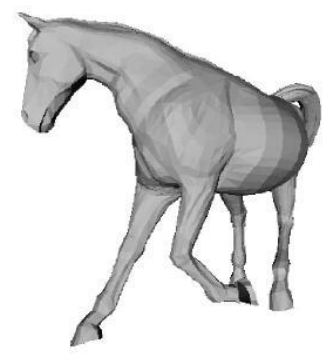

(c)

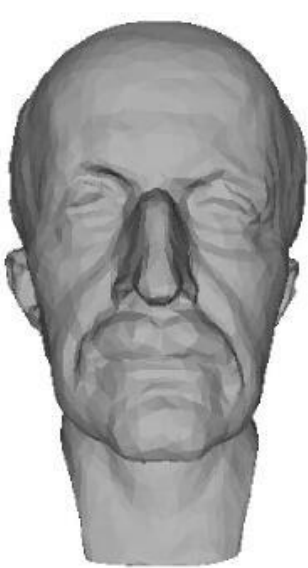

(d)

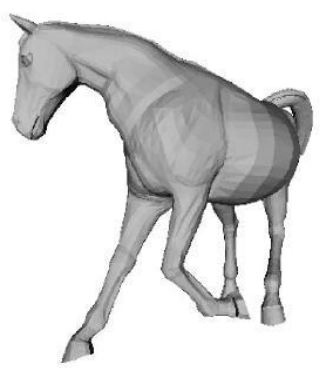

(d)

\section{Figure 3. Selected Results and their Corresponding Simplified Models Based on different Algorithms}

We apply our algorithm, Rossignac's clustering algorithm and Garland's QEM algorithm to simplify the three models in Figure 4 respectively. The results show by Figure 4. The first column is original models, the second and third columns are the results of using the clustering algorithm and QEM, the last column is based on our algorithm.

Like this paper, Rossignac's algorithm also subdivided the model by bounding box, so its simplified degree of each part is the same and visual effect looks evenly. From the experimental results we may see that the algorithm can guarantee the profile of original models, but it also lost many prominent local details, especially in some important feature positions of face. For example, both the eyes position of the first model and the upper half of the second model have insufficient visual performances.

Due to apply the QEM to control the simplified error, Garland's algorithm improved the details performance. However, when the simple error control strategy simplifies the model more in depth, it may cause the excess simplification and distorted effect, especially concentrated in the positions which have a lot of characteristics. Such as the hairs and mouth crack of the first model. There are great 
difference between the original model and simplified model.

On one side, this paper draws on the experience of advantages which in the two algorithms above, and also combines with the principle curvature, on the other side, we let the bounding box intersect with QEM after taking their defects into consideration. It not only makes the model simplified evenly, but also preserves its characteristics and controls the simplification degree effectively. The experimental results show that, our method is much better than the other two in handling details and holding the whole algorithm. The simplified model is the most close to original model based on our algorithm.

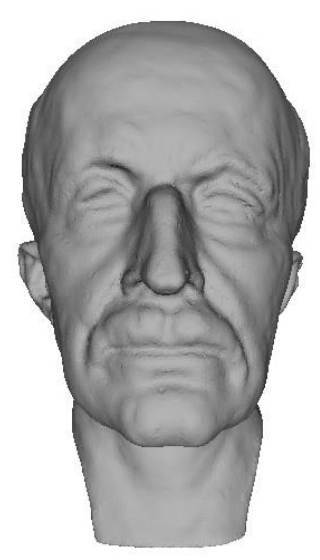

(a)

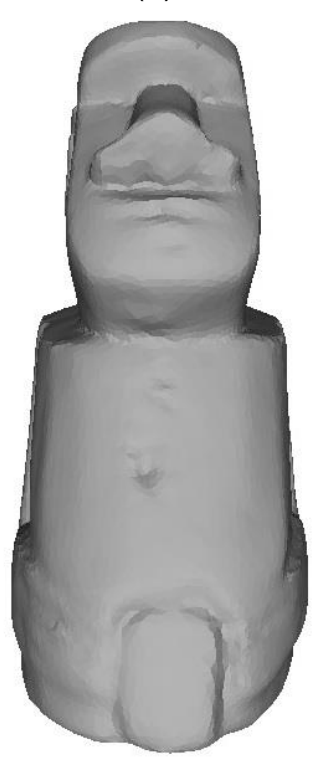

(a)

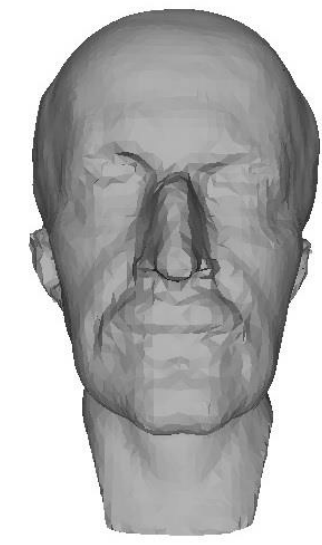

(b)

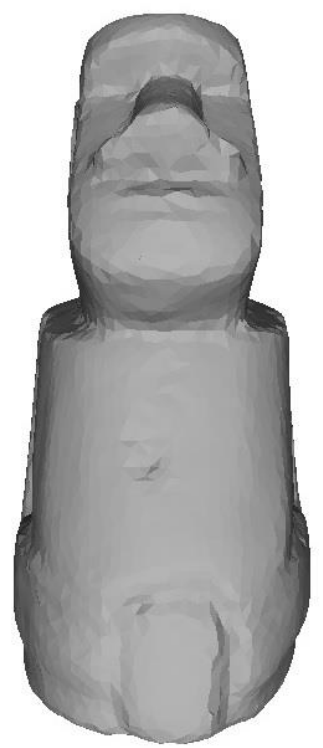

(b)

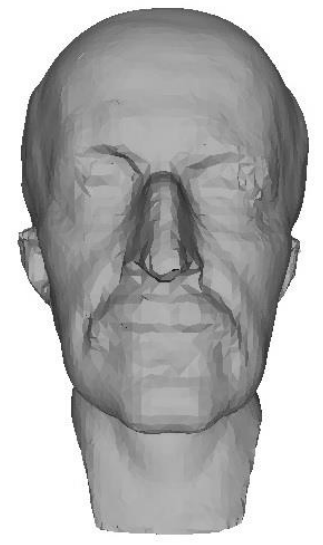

(c)

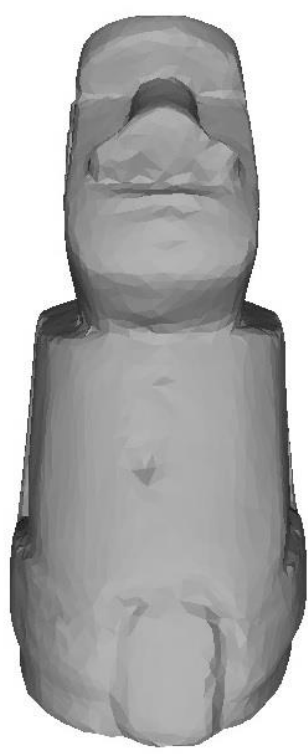

(c)

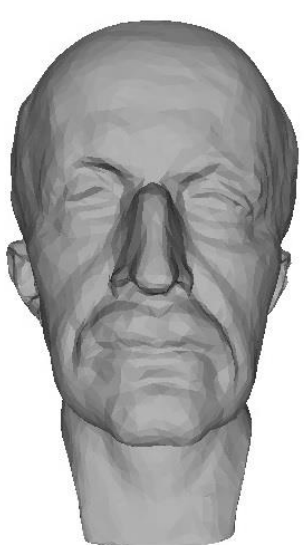

(d)

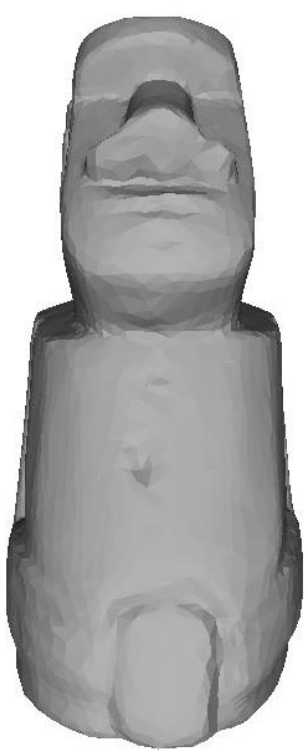

(d) 


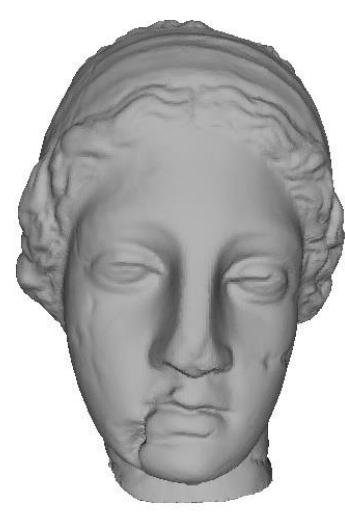

(a)

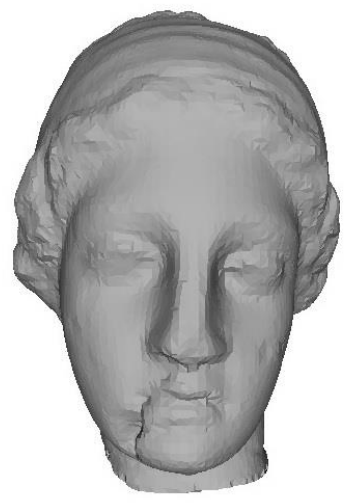

(b)

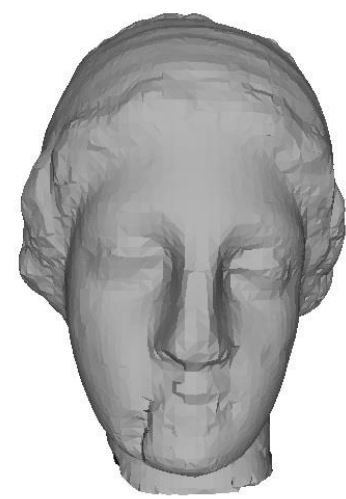

(c)

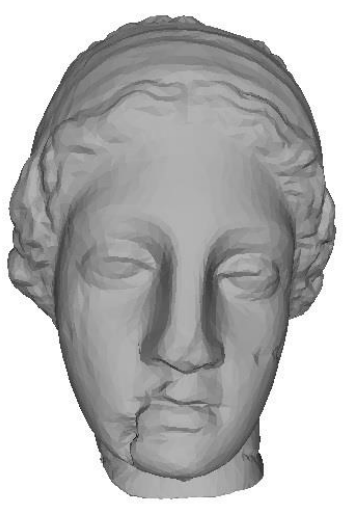

(d)

Figure 4. Several Experimental Results

\section{Conclusion}

This paper introduces a simplified algorithm using vertex clustering based on principal curvature, and combining the QEM with clustering. The algorithm only operates the vertices and triangles, don't involve the topological relation of too much vertices, line and surfaces. This trait provides the conditions for merging the triangles which geometry adjacency but topology-independent. The experiments prove: the algorithm removes vertices and triangles gradually. It also maintains the characteristics of original model at the greatest extent, especially for obvious characteristics but irregular model. The simplified model has high quality of reproduction.

The algorithm should pay attention to the following several aspects which need to improve in the future: we can adopt the inhomogeneous manner to subdivide the bounding box, so that the method may give consideration to feature information in different positions of the model, and make it without loss of generality. Moreover, although using the gradual simplified thinking, the error control method should still be perfected to increase the precision of whole algorithm.

\section{Acknowledgements}

Project supported by the National Nature Science Foundation of China (No. 61272430, 61173173, 61272245, 61472227); the Provincial Natural Science Foundation of Shandong under Grant No. ZR2013FM015.

\section{References}

[1] He H. G., Tian J., Zhang X. P., Zhao M. C. and Li G. M., "A Survey of Mesh Simplification”, vol. 12, no. 13, (2002).

[2] Luebke D., “A Developer's Survey of Polygonal Simplification Algorithms”, IEEE Computer Graphics \& Applications, vol. 3, no. 21, (2001).

[3] Rossignac J. and Borrel P., "Multi-Resolution 3D Approximation for Rendering Complex Scenes", In: Falcidieno, B. Kunii, T. L, eds. Proceedings of the 2nd Conference Modeling in Computer Graphics: 
Methods and Applications, Berlin, (1993).

[4] Zhou K., Pan Z. P. and Shi J. Y., "A New Mesh Simplification Algorithm Based on Vertex Clustering”, vol. 1 , no. 25, (1999).

[5] Turk G., "Re-tiling Polygonal Surface", vol. 2, no. 26, (1992).

[6] K. Zhou, X. H. Ma, Z. P. Pan and J. Y, Shi, "An Improved Algorithm of Triangle Mesh Simplification Based on Retiling", vol. 6, no. 9, (1998).

[7] Hoppe H., DeRose T., Duchamp T., McDonald J. and Stuetzle W., "Mesh Optimization", In: Proceedings of ACM SIGGRAPH, Anaheim, California, USA, (1993).

[8] Z. L. Tao, Z. P. Pan and J. Y. Shi, "Mesh Simplification Algorithm Based on Energy Evaluation and Its Application”, vol. 12, no. 8, (1997).

[9] R. R. Zhou, J. Tang, L. Y. Zhang and L. S. Zhou, "A Study on Mesh Simplification Algorithm Based on Controlling by Examining Ball”, vol. 11, no. 2, (2001).

[10] Kobbelt L., Campagna S. and Seidel H. P., "A General Framework for Mesh Decimation", In: Proceedings of the Graphics Interface, New York, (1998).

[11] Hamann B., “A Data Reduction Scheme for Triangulated Surfaces”, vol. 3, no. 11, (1994).

[12] Gieng T. S., Hamann B. and Joy K. I., "Smooth Hierarchical Surface Triangulations", In: Proceedings of the IEEE Visualization, Phoenix, Arizona, USA, (1997).

[13] X. H. Ma, Z. P. Pan and J. Y. Shi, "Polyhedral Model Simplicification Method Based on Triangle Removal Criterion", vol. 6, no. 21, (1998).

[14] Y F Zhou, C M Zhang and P He. Feature Preserving Mesh Simplification Algorithm Based on Square Volume Measure. 2, 32 (2009)

[15] Slmnir A., Bajaj C. and Pascucci V., "Multi-resolution Dynamic Meshes with Arbitrary Deformations", In: Proceedings of IEEE Visualization 2000 Conference Proceedings, Salt Lake City, Utah, (2000).

[16] Slmnir A. and Pascucci V., "Temporal and Spatial Level of Details for Dynamic Meshes", In: Proceedings of ACM Symposium on Virtual Reality Software and Technology, Alberta, (2001).

[17] Kircher S. and Garland M., "Progressive Multi-resolution Meshes for Deforming Surfaces", In: Proceedings of ACM S1GGRAPH/ Euro-graphics Symposium on Computer Animation, Los Angeles, California, (2005).

[18] F. C. Huang, B. Y. Chen and Y. Y. Chuang, "Progressive Deforming Meshes Based on Deformation Oriented Decimation and Dynamic Connectivity Updating”, In: Proceedings of ACM SIGGRAPH/Euro-graphics Symposium on Computer Animation, Vienna, (2006).

[19] S. M. Lv, M. L. Zhang and S. L. Sun, "Topological Optimization for Triangular Mesh Based on Simplification and Subdivision", vol. 8, no. 26, (2014).

[20] Cohen-Steiner D., Alliez P. and Desbrun M., "Variational Shape Approximation", In: Proceedings of the ACM SIGGRAPH, Los Angeles, (2004).

[21] W. Lu, D. H. Zeng and J. G. Pan, "Mesh Simplification for 3D Models with Feature-Preserving”, vol. 3, no. 20, (2009).

[22] L. Y. Zhang, R. R. Zhou, J. Tang and L. S. Zhou, "Research on Mesh Simplification with Additional Attributes”, vol. 3, no. 14, (2002).

[23] X. W. Liu, C. Xie and Y. C. Jin, “Appearance-Preserving Mesh Simplification”, vol. 11, no. 18, (2006).

[24] X. Feng and M. Q. Zhou, "An Algorithm for Simplification of 3D Models with Texture”, vol. 6, no. 21, (2009).

[25] Garland M. and P. S. Heckbert, "Surface Simplification Using Quadric Error Metrics”, In: Proceedings of the SIGGRAPH'97, Los Angeles, (1997).

[26] Pottmann H., Wallner J., Y. L. Yang, Y. K. Lai and S. M. Hu, "Principal Curvatures from the Integral Invariant Viewpoint”, vol. 8-9, no. 24, (2007).

[27] Y. P. Wang and S. M. Hu, “A New Watermarking Method for 3D Model Based on Integral Invariant”, IEEE Transactions on Visualization and Computer Graphics, vol. 2, no. 15, (2009). 